\title{
Exact Inverse Matrices of Fermat and Mersenne Circulant Matrix
}

\author{
Yanpeng Zheng and Sugoog Shon \\ Department of Information and Telecommunications Engineering, The University of Suwon, Wau-ri, Bongdam-eup, \\ Hwaseong-si, Gyeonggi-do 445-743, Republic of Korea
}

Correspondence should be addressed to Sugoog Shon; sshon@suwon.ac.kr

Received 25 July 2014; Accepted 17 September 2014

Academic Editor: Zidong Wang

Copyright (c) 2015 Y. Zheng and S. Shon. This is an open access article distributed under the Creative Commons Attribution License, which permits unrestricted use, distribution, and reproduction in any medium, provided the original work is properly cited.

\begin{abstract}
The well known circulant matrices are applied to solve networked systems. In this paper, circulant and left circulant matrices with the Fermat and Mersenne numbers are considered. The nonsingularity of these special matrices is discussed. Meanwhile, the exact determinants and inverse matrices of these special matrices are presented.
\end{abstract}

\section{Introduction}

Circulant matrices are an important tool in solving networked systems. In [1], the authors investigated the storage of binary cycles in Hopfield-type and other neural networks involving circulant matrix. In [2], the authors considered a special class of the feedback delay network using circulant matrices. Distributed differential space-time codes that work for networks with any number of relays using circulant matrices were proposed by Jing and Jafarkhani in [3]. Bašić [4] solved the question for when circulant quantum spin networks with nearest-neighbor couplings can give perfect state transfer. Wang et al. considered two-way transmission model ensured that circular convolution between two frequency selective channels in [5]. Li et al. [6] presented a lowcomplexity binary framewise network coding encoder design based on circulant matrix.

Circulant matrices have been applied to various disciplines including image processing, communications, signal processing, and encoding. Circulant type matrices have established the substantial basis with the work in [7-12] and so on.

Lately, some authors gave the explicit determinant and inverse of the circulant and skew-circulant involving famous numbers. For example, Yao and Jiang [13] presented the determinants, inverses, norm, and spread of skew circulant type matrices involving any continuous Lucas numbers.
Jiang et al. [14] considered circulant type matrices with the $k$-Fibonacci and $k$-Lucas numbers and presented the explicit determinant and inverse matrix by constructing the transformation matrices. Dazheng [15] got the determinant of the Fibonacci-Lucas quasi-cyclic matrices. Determinants and inverses of circulant matrices with Jacobsthal and JacobsthalLucas numbers were obtained by Bozkurt and Tam in [16].

For any integer $m \geq 0$, let $F_{m}=2^{2^{m}}+1$ be the $m$ th Fermat number. It is well known that $F_{m}$ is prime for $m \leq 4$, but there is no other $\mathrm{m}$ for which $F_{m}$ is known to prime. The Mersenne and Fermat sequences are defined by the following recurrence relations $[17,18]$, respectively:

$$
\begin{aligned}
\mathbb{M}_{n+1} & =3 \mathbb{M}_{n}-2 \mathbb{M}_{n-1} \\
\mathbb{F}_{n+1} & =3 \mathbb{F}_{n}-2 \mathbb{F}_{n-1}
\end{aligned}
$$

with the initial condition $\mathbb{M}_{0}=0, \mathbb{M}_{1}=1, \mathbb{F}_{0}=2, \mathbb{F}_{1}=3$, for $n \geq 1$.

Let $\alpha$ and $\beta$ be the roots of the characteristic equation $x^{2}-$ $3 x+2=0$; then the Binet formulas of the sequences $\left\{\mathbb{M}_{k+n}\right\}$ and $\left\{\mathbb{F}_{k+n}\right\}$ have the form

$$
\begin{gathered}
\mathbb{M}_{k+n}=\frac{\alpha^{k+n}-\beta^{k+n}}{\alpha-\beta}, \\
\mathbb{F}_{k+n}=\alpha^{k+n}+\beta^{k+n} .
\end{gathered}
$$


Lemma 1. Let $\mathbb{M}_{k+n}$ be the $(k+n)$ th Mersenne number and let $\mathbb{F}_{k+n}$ be the $(k+n)$ th Fermat number; then

(1) $\mathbb{M}_{n+1}-\mathbb{M}_{n}=2^{n}$

$\mathbb{M}_{n+1}-2 \mathbb{M}_{n}=1$,

$\mathbb{M}_{n}=2^{n}-1$,

$\mathbb{M}_{n}^{2}-\mathbb{M}_{n+1} \mathbb{M}_{n-1}=2^{n-1}$.

(2) $\mathbb{F}_{n+1}-\mathbb{F}_{n}=2^{n}$,

$\mathbb{F}_{n+1}-2 \mathbb{F}_{n}=-1$,

$\mathbb{F}_{n}=2^{n}+1$,

$\mathbb{F}_{n}^{2}-\mathbb{F}_{n+1} \mathbb{F}_{n-1}=-2^{n-1}$.

We define a Fermat circulant matrix which is an $n \times n$ matrix with the following form:

$$
\begin{aligned}
\operatorname{Circ} & \left(\mathbb{F}_{k+1}, \mathbb{F}_{k+2}, \ldots, \mathbb{F}_{k+n}\right) \\
= & {\left[\begin{array}{cccc}
\mathbb{F}_{k+1} & \mathbb{F}_{k+2} & \cdots & \mathbb{F}_{k+n} \\
\mathbb{F}_{k+n} & \mathbb{F}_{k+1} & \cdots & \mathbb{F}_{k+n-1} \\
\vdots & \vdots & & \vdots \\
\mathbb{F}_{k+2} & \mathbb{F}_{k+3} & \cdots & \mathbb{F}_{k+1}
\end{array}\right] . }
\end{aligned}
$$

A Mersenne circulant matrix which is an $n \times n$ matrix is defined with the following form:

$$
\begin{aligned}
\operatorname{Circ}\left(\mathbb{M}_{k+1}, \mathbb{M}_{k+2}, \ldots, \mathbb{M}_{k+n}\right) \\
=\left[\begin{array}{cccc}
\mathbb{M}_{k+1} & \mathbb{M}_{k+2} & \cdots & \mathbb{M}_{k+n} \\
\mathbb{M}_{k+n} & \mathbb{M}_{k+1} & \cdots & \mathbb{M}_{k+n-1} \\
\vdots & \vdots & & \vdots \\
\mathbb{M}_{k+2} & \mathbb{M}_{k+3} & \cdots & \mathbb{M}_{k+1}
\end{array}\right] .
\end{aligned}
$$

Besides, a Fermat left circulant matrix is given by

$$
\begin{aligned}
\operatorname{LCirc} & \left(\mathbb{F}_{k+1}, \mathbb{F}_{k+2}, \ldots, \mathbb{F}_{k+n}\right) \\
= & {\left[\begin{array}{cccc}
\mathbb{F}_{k+1} & \mathbb{F}_{k+2} & \cdots & \mathbb{F}_{k+n} \\
\mathbb{F}_{k+2} & \mathbb{F}_{k+3} & \cdots & \mathbb{F}_{k+1} \\
\vdots & \vdots & & \vdots \\
\mathbb{F}_{k+n} & \mathbb{F}_{k+1} & \cdots & \mathbb{F}_{k+n-1}
\end{array}\right] . }
\end{aligned}
$$

A Mersenne left circulant matrix is given by

$$
\begin{aligned}
\operatorname{LCirc} & \left(\mathbb{M}_{k+1}, \mathbb{M}_{k+2}, \ldots, \mathbb{M}_{k+n}\right) \\
= & {\left[\begin{array}{cccc}
\mathbb{M}_{k+1} & \mathbb{M}_{k+2} & \cdots & \mathbb{M}_{k+n} \\
\mathbb{M}_{k+2} & \mathbb{M}_{k+3} & \cdots & \mathbb{M}_{k+1} \\
\vdots & \vdots & & \vdots \\
\mathbb{M}_{k+n} & \mathbb{M}_{k+1} & \cdots & \mathbb{M}_{k+n-1}
\end{array}\right] . }
\end{aligned}
$$

The main content of this paper is to obtain the results for the exact determinants and inverses of Fermat and Mersenne circulant matrix. In this paper, let $k$ be a nonnegative integer, $A_{k, n}=\operatorname{Circ}\left(\mathbb{F}_{k+1}, \mathbb{F}_{k+2}, \ldots, \mathbb{F}_{k+n}\right)$, and $B_{k, n}=$ $\operatorname{Circ}\left(\mathbb{M}_{k+1}, \mathbb{M}_{k+2}, \ldots, \mathbb{M}_{k+n}\right)$.

\section{Determinant and Inverse of Fermat Circulant Matrix}

In this section, let $A_{k, n}=\operatorname{Circ}\left(\mathbb{F}_{k+1}, \mathbb{F}_{k+2}, \ldots, \mathbb{F}_{k+n}\right)$ be a Fermat circulant matrix. Firstly, we obtain the exact form determinant of the matrix $A_{k, n}$. Afterwards, we find the exact form inverse of the matrix $A_{k, n}$.

Theorem 2. Let $A_{k, n}=\operatorname{Circ}\left(\mathbb{F}_{k+1}, \mathbb{F}_{k+2}, \ldots, \mathbb{F}_{k+n}\right)$ be a Fermat circulant matrix. Then one has

$$
\begin{aligned}
\operatorname{det} A_{k, n}=\mathbb{F}_{k+1} \cdot & {\left[\sum_{j=1}^{n-2}\left(\mathbb{F}_{j+k+2}-\tau_{k} \mathbb{F}_{j+k+1}\right) \cdot y^{n-j-1}\right.} \\
& \left.+\mathbb{F}_{k+1}-\tau_{k} \mathbb{F}_{k+n}\right] \cdot(-f)^{n-2},
\end{aligned}
$$

where $y=-e / f, e=2\left(\mathbb{F}_{k}-\mathbb{F}_{k+n}\right), f=\mathbb{F}_{k+n+1}-\mathbb{F}_{k+1}, \tau_{k}=$ $\mathbb{F}_{k+2} / \mathbb{F}_{k+1}$, and $\mathbb{F}_{k+n}$ is the $(k+n)$ th Fermat number. Moreover, $A_{k, n}$ is singular if and only if $\left(1-\alpha \kappa_{l}\right)\left(1-\beta \kappa_{l}\right) \neq 0$ and $\mathbb{F}_{k+1}-$ $2 \kappa_{l} \mathbb{F}_{k}-\mathbb{F}_{k+n+1}+2 \kappa_{l} \mathbb{F}_{k+n}=0$, for $k \in N, n \in N_{+}$, where $\kappa_{l}=$ $\cos (2 l \pi / n)+i \sin (2 l \pi / n), l=1,2, \ldots, n$.

Proof. It is clear that det $A_{0, n}=\mathbb{F}_{1} \cdot\left[\sum_{j=1}^{n-2}\left(\mathbb{F}_{j+2}-\tau_{0} \mathbb{F}_{j+1}\right)\left[2\left(\mathbb{F}_{n}-\right.\right.\right.$ $\left.\left.\left.\mathbb{F}_{0}\right) /\left(\mathbb{F}_{n+1}-\mathbb{F}_{1}\right)\right]^{n-j-1}+\mathbb{F}_{1}-\tau_{0} \mathbb{F}_{n}\right] \cdot\left[\mathbb{F}_{1}-\mathbb{F}_{n+1}\right]^{n-2} \operatorname{satisfies}(7)$. In the following, let

$$
\Sigma=\left(\begin{array}{ccccccccc}
1 & & & & & & \\
-\tau_{k} & & & & & & 1 \\
2 & & & & & 1 & -3 \\
0 & & 0 & & 1 & -3 & 2 \\
\vdots & & & & . & . & . & \\
0 & & & 1 & . & . & & \\
0 & & 1 & -3 & . & & 0 & \\
0 & 1 & -3 & 2 & & & &
\end{array}\right)
$$

$$
\Omega_{1}=\left(\begin{array}{cccccc}
1 & 0 & 0 & \cdots & 0 & 0 \\
0 & y^{n-2} & 0 & \cdots & 0 & 0 \\
0 & y^{n-3} & 0 & \cdots & 0 & -1 \\
\vdots & \vdots & \vdots & \ddots & \vdots & \vdots \\
0 & y & 0 & \cdots & 0 & 0 \\
0 & 1 & -1 & \cdots & 0 & 0
\end{array}\right)
$$

be two $n \times n$ matrices; we have

$$
\sum A_{k, n} \Omega_{1}=\left(\begin{array}{cccccc}
\mathbb{F}_{k+1} & h_{k, n}^{\prime} & -\mathbb{F}_{k+n} & -\mathbb{F}_{k+n-1} & \cdots & -\mathbb{F}_{k+3} \\
0 & h_{k, n} & a_{3} & a_{4} & \cdots & a_{n} \\
0 & 0 & e & f & & 0 \\
0 & 0 & 0 & e & & 0 \\
\vdots & \vdots & \vdots & \vdots & \ddots & \vdots \\
0 & 0 & 0 & 0 & \ddots & f \\
0 & 0 & 0 & 0 & & e
\end{array}\right) \text {, }
$$


where

$$
\begin{gathered}
\tau_{k}=\frac{\mathbb{F}_{k+2}}{\mathbb{F}_{k+1}}, \\
y=-\frac{e}{f}, \\
a_{3}=\tau_{k} \mathbb{F}_{k+n}-\mathbb{F}_{k+1}, \\
a_{j}=\tau_{k} \mathbb{F}_{k+n+3-j}-\mathbb{F}_{k+n+4-j} \\
(j=4,5, \ldots, n), \\
h_{k, n}^{\prime}=\sum_{t=1}^{n-1} \mathbb{F}_{t+k+1}\left[\frac{2\left(\mathbb{F}_{k+n}-\mathbb{F}_{k}\right)}{\mathbb{F}_{k+n+1}-\mathbb{F}_{k+1}}\right]^{n-t-1}, \\
h_{k, n}=\sum_{t=1}^{n-2}\left(\mathbb{F}_{t+k+2}-\tau_{k} \mathbb{F}_{t+k+1}\right) y^{n-t-1} \\
+\mathbb{F}_{k+1}-\tau_{k} \mathbb{F}_{k+n} .
\end{gathered}
$$

We obtain

$$
\begin{aligned}
& \operatorname{det} \Sigma \operatorname{det} A_{k, n} \operatorname{det} \Omega_{1} \\
&=\mathbb{F}_{k+1} \cdot\left[\sum_{t=1}^{n-2}\left(\mathbb{F}_{t+k+2}-\tau_{k} \mathbb{F}_{t+k+1}\right) y^{n-t-1}\right. \\
&\left.\quad+\mathbb{F}_{k+1}-\tau_{k} \mathbb{F}_{k+n}\right] \cdot e^{n-2},
\end{aligned}
$$

while

$$
\begin{gathered}
\operatorname{det} \Sigma=(-1)^{(n-1)(n-2) / 2} \\
\operatorname{det} \Omega_{1}=(-1)^{(n-1)(n-2) / 2}\left[\frac{2\left(\mathbb{F}_{k}-\mathbb{F}_{k+n}\right)}{\mathbb{F}_{k+1}-\mathbb{F}_{k+n+1}}\right]^{n-2} .
\end{gathered}
$$

We have

$$
\begin{aligned}
\operatorname{det} A_{k, n}= & \mathbb{F}_{k+1} \\
& \quad\left[\sum_{t=1}^{n-2}\left(\mathbb{F}_{t+k+2}-\tau_{k} \mathbb{F}_{t+k+1}\right) \cdot y^{n-t-1}\right. \\
& \left.\quad+\mathbb{F}_{k+1}-\tau_{k} \mathbb{F}_{k+n}\right] \cdot(-f)^{n-2} .
\end{aligned}
$$

Next, we discuss the singularity of the matrix $A_{k, n}$.

The roots of polynomial $g(x)=x^{n}-1$ are $\kappa_{l}(l=$ $1,2, \ldots, n)$, where $\kappa_{l}=\cos (2 l \pi / n)+i \sin (2 l \pi / n)$. We have

$$
\begin{aligned}
f\left(\kappa_{l}\right) & =\mathbb{F}_{k+1}+\mathbb{F}_{k+2} \kappa_{l}+\cdots+\mathbb{F}_{k+n}\left(\kappa_{l}\right)^{n-1} \\
& =\frac{\mathbb{F}_{k+1}-2 \kappa_{l} \mathbb{F}_{k}-\mathbb{F}_{k+n+1}+2 \kappa_{l} \mathbb{F}_{k+n}}{\left(1-\alpha \kappa_{l}\right)\left(1-\beta \kappa_{l}\right)} .
\end{aligned}
$$

By Lemma 1 in [14], the matrix $A_{k, n}$ is nonsingular if and only if $f\left(\kappa_{l}\right) \neq 0$; that is, when $\left(1-\alpha \kappa_{l}\right)\left(1-\beta \kappa_{l}\right) \neq 0, A_{k, n}$ is nonsingular if and only if $\mathbb{F}_{k+1}-2 \kappa_{l} \mathbb{F}_{k}-\mathbb{F}_{k+n+1}+2 \kappa_{l} \mathbb{F}_{k+n} \neq 0$; when $\left(1-\alpha \kappa_{l}\right)\left(1-\beta \kappa_{l}\right)=0$, we obtain $\kappa_{l}=1 / \alpha$ or $\kappa_{l}=1 / \beta$.

Let $\kappa_{l}=1 / \alpha$; then the eigenvalue of $A_{k, n}$ is

$$
f\left(\kappa_{l}\right)=\frac{n \alpha^{k+n}-\beta^{k+1} \mathbb{F}_{n}}{\alpha^{n-1}(\alpha-\beta)} \neq 0,
$$

for $\alpha=2, \beta=1, k \in N, n \in N_{+}, l=1,2, \ldots, n$, so $A_{k, n}$ is nonsingular. The arguments for $\kappa_{l}=1 / \beta$ are similar. Thus, the proof is completed.

Lemma 3. Let the matrix $\mathfrak{M}=\left[m_{i, l}^{\prime}\right]_{i, l=1}^{n-2}$ be of the form

$$
m_{i, l}= \begin{cases}2\left(\mathbb{F}_{k}-\mathbb{F}_{k+n}\right)=e, & i=l, \\ \mathbb{F}_{k+n+1}-\mathbb{F}_{k+1}=f, & l=i+1, \\ 0, & \text { otherwise. }\end{cases}
$$

Then the inverse $\mathfrak{M}^{-1}=\left[m_{i, l}^{\prime}\right]_{i, l=1}^{n-2}$ of the matrix $\mathfrak{M}$ is equal to

$$
m_{i, l}^{\prime}= \begin{cases}\frac{\left(\mathbb{F}_{k+1}-\mathbb{F}_{k+n+1}\right)^{l-i}}{\left[2\left(\mathbb{F}_{k}-\mathbb{F}_{k+n}\right)\right]^{l-i+1}}=\frac{(-f)^{l-i}}{e^{l-i+1}}, & l \geq i, \\ 0, & l<i .\end{cases}
$$

Proof. Let $e_{i, l}=\sum_{k=1}^{n-2} m_{i, k} m_{k, l}^{\prime}$. Distinctly, $c_{i, l}=0$ for $l<i$. In the case $i=l$, we obtain

$$
\begin{aligned}
e_{i, i} & =m_{i, i} m_{i, i}^{\prime} \\
& =\left(\mathbb{F}_{k+1}-\mathbb{F}_{k+n+1}\right) \cdot \frac{1}{\left(\mathbb{F}_{k+1}-\mathbb{F}_{k+n+1}\right)} \\
& =1 .
\end{aligned}
$$

For $l \geq i+1$, we get

$$
\begin{aligned}
e_{i, l} & =\sum_{k=1}^{n-2} m_{i, k} m_{k, l}^{\prime} \\
& =m_{i, i} m_{i, l}^{\prime}+m_{i, i+1} m_{i+1, l}^{\prime} \\
& =e \cdot \frac{(-f)^{l-i}}{e^{l-i+1}}+f \cdot \frac{(-f)^{l-i-1}}{e^{l-i}} \\
& =0 .
\end{aligned}
$$

We check on $\mathfrak{M M}^{-1}=I_{n-2}$, where $I_{n-2}$ is $(n-2) \times(n-2)$ identity matrix. Similarly, we can verify $\mathfrak{M}^{-1} \mathfrak{M}=I_{n-2}$. Thus, the proof is completed. 
Theorem 4. Let $A_{k, n}=\operatorname{Circ}\left(\mathbb{F}_{k+1}, \mathbb{F}_{k+2}, \ldots, \mathbb{F}_{k+n}\right)$ be a Fermat circulant matrix. Then one acquires $A_{k, n}^{-1}=\operatorname{Circ}\left(v_{1}, v_{2}, \ldots\right.$, $\left.v_{n}\right)$, where

$$
\begin{aligned}
& v_{1}=\frac{1}{h_{k, n}}+\left(\mathbb{F}_{k+1}-\tau_{k} \mathbb{F}_{k+n}-h_{k, n}\right) \\
& \cdot \frac{-\mathbb{F}_{k+n+1}+3 \mathbb{F}_{k+n}+\mathbb{F}_{k+1}-3 \mathbb{F}_{k}}{2 h_{k, n}\left(\mathbb{F}_{k}-\mathbb{F}_{k+n}\right)^{2}} \\
& +\frac{\left(\mathbb{F}_{k+n}-\tau_{k} \mathbb{F}_{k+n-1}\right)}{h_{k, n}\left(\mathbb{F}_{k}-\mathbb{F}_{k+n}\right)}, \\
& v_{2}=\frac{-2^{k}-h_{k, n} \mathbb{F}_{k+1}}{\mathbb{F}_{k+1} h_{k, n}\left(\mathbb{F}_{k}-\mathbb{F}_{k+n}\right)}, \\
& v_{3}=\frac{\mathbb{F}_{k+1}-\tau_{k} \mathbb{F}_{k+n}-h_{k, n}}{h_{k, n}} \\
& \cdot \frac{\left(\mathbb{F}_{k+1}-\mathbb{F}_{k+n+1}\right)^{n-3}}{\left[2\left(\mathbb{F}_{k}-\mathbb{F}_{k+n}\right)\right]^{n-2}}+\frac{1}{h_{k, n}} \\
& \cdot \sum_{i=4}^{n}\left(\mathbb{F}_{k+n+4-i}-\tau_{k} \mathbb{F}_{k+n+3-i}\right) \\
& \cdot \frac{\left(\mathbb{F}_{k+1}-\mathbb{F}_{k+n+1}\right)^{n-i}}{\left[2\left(\mathbb{F}_{k}-\mathbb{F}_{k+n}\right)\right]^{n-i+1}}, \\
& v_{4}=\frac{\mathbb{F}_{k+n+2}-\mathbb{F}_{k+2}}{h_{k, n}} \\
& \times\left[\left(\mathbb{F}_{k+1}-\tau_{k} \mathbb{F}_{k+n}-h_{k, n}\right)\right. \\
& \times \frac{\left(\mathbb{F}_{k+1}-\mathbb{M}_{k+n+1}\right)^{n-4}}{\left[2\left(\mathbb{M}_{k}-\mathbb{M}_{k+n}\right)\right]^{n-2}} \\
& +\sum_{i=4}^{n}\left(\mathbb{F}_{k+n+4-i}-\tau_{k} \mathbb{F}_{k+n+3-i}\right) \\
& \left.\cdot \frac{\left(\mathbb{F}_{k+1}-\mathbb{F}_{k+n+1}\right)^{n-i-1}}{\left[2\left(\mathbb{F}_{k}-\mathbb{F}_{k+n}\right)\right]^{n-i+1}}\right], \\
& v_{s}=0 \quad(s=5,6, \ldots, n) \text {. }
\end{aligned}
$$

Proof. Let

$$
\Omega_{2}=\left(\begin{array}{cccccc}
1 & -\frac{h_{k, n}^{\prime}}{\mathbb{F}_{k+1}} & x_{3}^{\prime} & x_{4}^{\prime} & \cdots & x_{n}^{\prime} \\
0 & 1 & y_{3}^{\prime} & y_{4}^{\prime} & \cdots & y_{n}^{\prime} \\
0 & 0 & 1 & 0 & \cdots & 0 \\
0 & 0 & 0 & 1 & \cdots & 0 \\
\vdots & \vdots & \vdots & \vdots & \ddots & \vdots \\
0 & 0 & 0 & 0 & \cdots & 1
\end{array}\right),
$$

where

$$
\begin{gathered}
\tau_{k}=\frac{\mathbb{F}_{k+2}}{\mathbb{F}_{k+1}}, \\
x_{3}^{\prime}=\frac{\mathbb{F}_{k+n}}{\mathbb{F}_{k+1}}+\frac{h_{k, n}^{\prime}}{h_{k, n}} \cdot \frac{\left(\tau_{k} \mathbb{F}_{k+n}-\mathbb{F}_{k+1}\right)}{\mathbb{F}_{k+1}}, \\
y_{3}^{\prime}=\frac{\mathbb{F}_{k+1}-\tau_{k} \mathbb{F}_{k+n}}{h_{k, n}}, \\
x_{i}^{\prime}=\frac{\mathbb{F}_{k+n+3-i}+\frac{h_{k, n}^{\prime}}{h_{k, n}}}{\mathbb{F}_{k+1}} \\
\frac{\tau_{k} \mathbb{F}_{k+n+3-i}-\mathbb{F}_{k+n+4-i}}{\mathbb{F}_{k+1}} \quad(i=4, \ldots, n), \\
y_{i}^{\prime}=\frac{\mathbb{F}_{k+n+4-i}-\tau_{k} \mathbb{F}_{k+n+3-i}}{h_{k, n}} \quad(i=4, \ldots, n), \\
h_{k, n}^{\prime}=\sum_{i=1}^{n-1} \mathbb{F}_{i+k+1}\left[\frac{2\left(\mathbb{F}_{k+n}-\mathbb{F}_{k}\right)}{\mathbb{F}_{k+n+1}-\mathbb{F}_{k+1}}\right]^{n-i-1}, \\
h_{k, n}=\sum_{i=1}^{n-2}\left(\mathbb{F}_{i+k+2}-\tau_{k} \mathbb{F}_{i+k+1}\right) y^{n-i-1},
\end{gathered}
$$

We have

$$
\sum A_{k, n} \Omega_{1} \Omega_{2}=\mathscr{D}_{2} \oplus \mathfrak{M},
$$

where $\mathscr{D}_{2}=\operatorname{diag}\left(\mathbb{F}_{k+1}, h_{k, n}\right)$ is a diagonal matrix, and $\mathscr{D}_{2} \oplus \mathfrak{M}$ is the direct sum of $\mathscr{D}_{2}$ and $\mathfrak{M}$. If we denote $\Omega=\Omega_{1} \Omega_{2}$, then we obtain

$$
A_{k, n}^{-1}=\Omega\left(\mathscr{D}_{2}^{-1} \oplus \mathfrak{M}^{-1}\right) \Sigma
$$

Let $A_{k, n}^{-1}=\operatorname{Circ}\left(v_{1}, v_{2}, \ldots, v_{n}\right)$. Since the last row elements of the matrix $\Omega$ are $0,1, y_{3}^{\prime}-1, y_{4}^{\prime}, \ldots, y_{n-1}^{\prime}, y_{n}^{\prime}$, according to Lemma 3 , then the last row elements of $A_{k, n}^{-1}$ are given by the following equations:

$$
\begin{aligned}
& v_{2}=-\frac{\tau_{k}}{h_{k, n}}+\frac{y_{3}^{\prime}-1}{\mathbb{F}_{k}-\mathbb{F}_{k+n}}, \\
& v_{3}=\left(y_{3}^{\prime}-1\right) \frac{(-f)^{n-3}}{e^{n-2}}+\sum_{i=4}^{n} y_{i}^{\prime} \cdot \frac{(-f)^{n-i}}{e^{n-i+1}}, \\
& v_{4}=\left(y_{3}^{\prime}-1\right)\left[\frac{(-f)^{n-4}}{(-f)^{n-3}}-\frac{3(-f)^{n-3}}{e^{n-2}}\right] \\
&+\sum_{i=4}^{n} y_{i}^{\prime} \cdot\left[\frac{(-f)^{n-i-1}}{e^{n-i}}-\frac{3(-f)^{n-i}}{e^{n-i+1}}\right] \\
&\left(t<0,(-f)^{t}=0\right),
\end{aligned}
$$




$$
\begin{gathered}
v_{s}=\left(y_{3}^{\prime}-1\right)\left[\frac{(-f)^{n-s}}{e^{n-s+1}}-\frac{3(-f)^{n-s+1}}{e^{n-s+2}}+\frac{2(-f)^{n-s+2}}{e^{n-s+3}}\right] \\
+\sum_{i=4}^{n-s+5} y_{i}^{\prime} \cdot\left[\frac{(-f)^{n-i-s+3}}{e^{n-i-s+4}}-\frac{3(-f)^{n-i-s+4}}{e^{n-i-s+5}}\right. \\
\left.+\frac{2(-f)^{n-i-s+5}}{e^{n-i-s+6}}\right] \\
\left(s=5,6, \ldots, n ; t<0,(-f)^{t}=0\right), \\
v_{1}=\frac{1}{h_{k, n}}+\frac{-2 f-3 e}{e^{2}}\left(y_{3}-1\right)+\frac{2}{e} y_{4},
\end{gathered}
$$

where $f=\mathbb{F}_{k+n+1}-\mathbb{F}_{k+1}, e=2\left(\mathbb{F}_{k}-\mathbb{F}_{k+n}\right)$, according to Lemma 1; then we have

(i) $e+f=0$,

(ii) $e+2 f=2^{k+n+1}-2^{k+1}$.

Hence, we obtain

$$
\begin{aligned}
& v_{1}=\frac{1}{h_{k, n}}+\left(\mathbb{F}_{k+1}-\tau_{k} \mathbb{F}_{k+n}-h_{k, n}\right) \\
& \cdot \frac{-\mathbb{F}_{k+n+1}+3 \mathbb{F}_{k+n}+\mathbb{F}_{k+1}-3 \mathbb{F}_{k}}{2 h_{k, n}\left(\mathbb{F}_{k}-\mathbb{F}_{k+n}\right)^{2}} \\
& +\frac{\left(\mathbb{F}_{k+n}-\tau_{k} \mathbb{F}_{k+n-1}\right)}{h_{k, n}\left(\mathbb{F}_{k}-\mathbb{F}_{k+n}\right)}, \\
& v_{2}=\frac{-2^{k}-h_{k, n} \mathbb{F}_{k+1}}{\mathbb{F}_{k+1} h_{k, n}\left(\mathbb{F}_{k}-\mathbb{F}_{k+n}\right)}, \\
& v_{3}=\frac{\mathbb{F}_{k+1}-\tau_{k} \mathbb{F}_{k+n}-h_{k, n}}{h_{k, n}} \\
& \cdot \frac{\left(\mathbb{F}_{k+1}-\mathbb{F}_{k+n+1}\right)^{n-3}}{\left[2\left(\mathbb{F}_{k}-\mathbb{F}_{k+n}\right)\right]^{n-2}}+\frac{1}{h_{k, n}} \\
& \cdot \sum_{i=4}^{n}\left(\mathbb{F}_{k+n+4-i}-\tau_{k} \mathbb{F}_{k+n+3-i}\right) \\
& \cdot \frac{\left(\mathbb{F}_{k+1}-\mathbb{F}_{k+n+1}\right)^{n-i}}{\left[2\left(\mathbb{F}_{k}-\mathbb{F}_{k+n}\right)\right]^{n-i+1}}, \\
& v_{4}=\frac{\mathbb{F}_{k+n+2}-\mathbb{F}_{k+2}}{h_{k, n}} \\
& \times\left[\left(\mathbb{F}_{k+1}-\tau_{k} \mathbb{F}_{k+n}-h_{k, n}\right)\right. \\
& \cdot \frac{\left(\mathbb{F}_{k+1}-\mathbb{F}_{k+n+1}\right)^{n-4}}{\left[2\left(\mathbb{F}_{k}-\mathbb{F}_{k+n}\right)\right]^{n-2}}
\end{aligned}
$$

$$
\begin{array}{r}
+\sum_{i=4}^{n}\left(\mathbb{F}_{k+n+4-i}-\tau_{k} \mathbb{F}_{k+n+3-i}\right) \\
\left.\times \frac{\left(\mathbb{F}_{k+1}-\mathbb{F}_{k+n+1}\right)^{n-i-1}}{\left[2\left(\mathbb{F}_{k}-\mathbb{F}_{k+n}\right)\right]^{n-i+1}}\right], \\
v_{s}=0 \quad(s=5,6, \ldots, n) .
\end{array}
$$

Thus, the proof is completed.

\section{Determinant and Inverse of Mersenne Circulant Matrix}

In this section, let $B_{k, n}=\operatorname{Circ}\left(\mathbb{M}_{k+1}, \mathbb{M}_{k+2}, \ldots, \mathbb{M}_{k+n}\right)$ be a Mersenne circulant matrix. Firstly, we obtain the determinant of the matrix $B_{k, n}$. Afterwards, we seek out the inverse of the matrix $B_{k, n}$.

Theorem 5. Let $B_{k, n}=\operatorname{Circ}\left(\mathbb{M}_{k+1}, \mathbb{M}_{k+2}, \ldots, \mathbb{M}_{k+n}\right)$ be a Mersenne circulant matrix. Then one obtains

$$
\begin{aligned}
\operatorname{det} B_{k, n}= & \mathbb{M}_{k+1} \\
& \cdot\left[\sum_{k=1}^{n-2}\left(\mathbb{M}_{k+k+2}-\mu_{k} \mathbb{M}_{k+k+1}\right) x^{n-k-1}\right. \\
& \left.+\mathbb{M}_{k+1}-\mu_{k} \mathbb{M}_{k+n}\right] \cdot(-d)^{n-2},
\end{aligned}
$$

where $x=-c / d, c=2\left(\mathbb{M}_{k}-\mathbb{M}_{k+n}\right), d=\mathbb{M}_{k+n+1}-\mathbb{M}_{k+1}$, $\mu_{k}=\mathbb{M}_{k+2} / \mathbb{M}_{k+1}$, and $\mathbb{M}_{k+n}$ is the $(k+n)$ th Mersenne number. Furthermore, $B_{k, n}$ is singular if and only if $\left(1-\alpha \kappa_{l}\right)\left(1-\beta \kappa_{l}\right) \neq 0$ and $\mathbb{M}_{k+1}-2 \kappa_{l} \mathbb{M}_{k}-\mathbb{M}_{k+n+1}+2 \kappa_{l} \mathbb{M}_{k+n}=0$, for $k \in N, n \in N_{+}$, where $\kappa_{l}=\cos (2 l \pi / n)+i \sin (2 l \pi / n), l=1,2, \ldots, n$.

Proof. Obviously,

$$
\begin{aligned}
\operatorname{det} A_{0, n}= & \mathbb{M}_{1} \\
& \cdot\left[\sum_{i=1}^{n-2}\left(\mathbb{M}_{i+2}-\mu_{0} \mathbb{M}_{i+1}\right) \cdot\left[\frac{2\left(\mathbb{M}_{n}-\mathbb{M}_{0}\right)}{\mathbb{M}_{n+1}-\mathbb{M}_{1}}\right]^{n-k-1}\right. \\
& \left.+\mathbb{M}_{1}-\mu_{0} \mathbb{M}_{n}\right] \cdot\left[2\left(\mathbb{M}_{0}-\mathbb{M}_{n}\right)\right]^{n-2}
\end{aligned}
$$


satisfies (27). In the following, let

$$
\Gamma=\left(\begin{array}{ccccccccc}
1 & & & & & & \\
-\mu_{k} & & & & & & 1 \\
2 & & & & & 1 & -3 \\
0 & & 0 & & 1 & -3 & 2 \\
\vdots & & & & . & . & . & \\
0 & & & 1 & . & \ddots & & \\
0 & & 1 & -3 & . & & 0 & \\
0 & 1 & -3 & 2 & & & &
\end{array}\right) \text {, }
$$

$$
\Pi_{1}=\left(\begin{array}{cccccc}
1 & 0 & 0 & \cdots & 0 & 0 \\
0 & x^{n-2} & 0 & \cdots & 0 & 0 \\
0 & x^{n-3} & 0 & \cdots & 0 & -1 \\
\vdots & \vdots & \vdots & \ddots & \vdots & \vdots \\
0 & x & 0 & \cdots & 0 & 0 \\
0 & 1 & -1 & \cdots & 0 & 0
\end{array}\right)
$$

be two $n \times n$ matrices; then we have

$$
\Gamma B_{k, n} \Pi_{1}=\left(\begin{array}{ccccc}
\mathbb{M}_{k+1} & f_{k, n}^{\prime} & -\mathbb{M}_{k+n} & \cdots & -\mathbb{M}_{k+3} \\
0 & f_{k, n} & h_{3} & \cdots & h_{n} \\
0 & 0 & c & \cdots & 0 \\
0 & 0 & 0 & \cdots & 0 \\
\vdots & \vdots & \vdots & \ddots & \vdots \\
0 & 0 & 0 & \cdots & d \\
0 & 0 & 0 & \cdots & c
\end{array}\right),
$$

where

$$
\begin{gathered}
c=2\left(\mathbb{M}_{k}-\mathbb{M}_{k+n}\right), \\
d=\mathbb{M}_{k+n+1}-\mathbb{M}_{k+1}, \\
x=-\frac{c}{d}, \quad \mu_{k}=\frac{\mathbb{M}_{k+2}}{\mathbb{M}_{k+1}}, \\
h_{3}=\mu_{k} \mathbb{M}_{k+n}-\mathbb{M}_{k+1}, \\
h_{j}=\left(\mu_{k} \mathbb{M}_{k+n+3-j}-\mathbb{M}_{k+n+4-j}\right) \\
f_{k, n}^{\prime}=\sum_{i=1}^{n-1} \mathbb{M}_{i+k+1}\left[\frac{2(j=4,5, \ldots, n),}{\mathbb{M}_{k+n+1}-\mathbb{M}_{k+1}}\right]^{n-i-1} \\
f_{k, n}=\sum_{i=1}^{n-2}\left(\mathbb{M}_{i+k+2}-\mu_{k} \mathbb{M}_{i+k+1}\right), x^{n-i-1} \\
+\mathbb{M}_{k+1}-\mu_{k} \mathbb{M}_{k+n} .
\end{gathered}
$$

We get

$$
\begin{aligned}
\operatorname{det} \Gamma \operatorname{det} B_{k, n} \operatorname{det} \Pi_{1} \\
=\mathbb{M}_{k+1} \cdot\left[\sum_{i=1}^{n-2}\left(\mathbb{M}_{i+k+2}-\mu_{k} \mathbb{M}_{i+k+1}\right) x^{n-i-1}\right. \\
\left.+\mathbb{M}_{k+1}-\mu_{k} \mathbb{M}_{k+n}\right] \cdot c^{n-2}
\end{aligned}
$$

besides

$$
\begin{gathered}
\operatorname{det} \Gamma=(-1)^{(n-1)(n-2) / 2}, \\
\operatorname{det} \Pi_{1}=(-1)^{(n-1)(n-2) / 2}\left[\frac{2\left(\mathbb{M}_{k+n}-\mathbb{M}_{k}\right)}{\mathbb{M}_{k+n+1}-\mathbb{M}_{k+1}}\right]^{n-2} .
\end{gathered}
$$

We have

$$
\begin{array}{r}
\operatorname{det} B_{k, n}=\mathbb{M}_{k+1} \cdot\left[\sum_{i=1}^{n-2}\left(\mathbb{M}_{i+k+2}-\mu_{k} \mathbb{M}_{i+k+1}\right) x^{n-i-1}\right. \\
\left.+\mathbb{M}_{k+1}-\mu_{k} \mathbb{M}_{k+n}\right] \cdot(-d)^{n-2} .
\end{array}
$$

Now, we discuss the singularity of the matrix $B_{k, n}$.

The roots of polynomial $g(x)=x^{n}-1$ are $\kappa_{l}(l=$ $1,2, \ldots, n)$, where $\kappa_{l}=\cos (2 l \pi / n)+i \sin (2 l \pi / n)$. So we have

$$
\begin{aligned}
f\left(\kappa_{l}\right) & =\mathbb{M}_{k+1}+\mathbb{M}_{k+2} \kappa_{l}+\cdots+\mathbb{M}_{k+n}\left(\kappa_{l}\right)^{n-1} \\
& =\frac{\mathbb{M}_{k+1}-2 \kappa_{l} \mathbb{M}_{k}-\mathbb{M}_{k+n+1}+2 \kappa_{l} \mathbb{M}_{k+n}}{\left(1-\alpha \kappa_{l}\right)\left(1-\beta \kappa_{l}\right)} .
\end{aligned}
$$

By Lemma 1 in [14], the matrix $B_{k, n}$ is nonsingular if and only if $f\left(\kappa_{l}\right) \neq 0$. That is when $\left(1-\alpha \kappa_{l}\right)\left(1-\beta \kappa_{l}\right) \neq 0, B_{k, n}$ is nonsingular if and only if $\mathbb{M}_{k+1}-2 \kappa_{l} \mathbb{M}_{k}-\mathbb{M}_{k+n+1}+2 \kappa_{l} \mathbb{M}_{k+n} \neq$ 0 , for $k \in N, n \in N_{+}, l=1,2, \ldots, n$. When $\left(1-\alpha \kappa_{l}\right)\left(1-\beta \kappa_{l}\right)=$ 0 , we obtain $\kappa_{l}=1 / \alpha$ or $\kappa_{l}=1 / \beta$. Let $\kappa_{l}=1 / \alpha$; then the eigenvalue of $B_{k, n}$ is

$$
f\left(\kappa_{l}\right)=\frac{n \alpha^{k+n}-\beta^{k+1} \mathbb{M}_{n}}{\alpha^{n-1}(\alpha-\beta)} \neq 0,
$$

for $\alpha=2, \beta=1, k \in N, n \in N_{+}, l=1,2, \ldots, n$, so $B_{k, n}$ is nonsingular. The arguments for $\kappa_{l}=1 / \beta$ are similar. Thus, the proof is completed.

Lemma 6. Let the matrix $\left(\mathfrak{G}=\left[g_{i, j}\right]_{i, j=1}^{n-2}\right.$ be of the form

$$
g_{i, j}= \begin{cases}2\left(\mathbb{M}_{k}-\mathbb{M}_{k+n}\right)=c, & i=j, \\ \mathbb{M}_{k+n+1}-\mathbb{M}_{k+1}=d, & j=i+1, \\ 0, & \text { otherwise. }\end{cases}
$$

Then the inverse $\mathfrak{G}^{-1}=\left[g_{i, j}^{\prime}\right]_{i, j=1}^{n-2}$ of the matrix $\mathfrak{G}$ is equal to

$$
g_{i, j}^{\prime}= \begin{cases}\frac{\left(\mathbb{M}_{k+1}-\mathbb{M}_{k+n+1}\right)^{j-i}}{\left[2\left(\mathbb{M}_{k}-\mathbb{M}_{k+n}\right)\right]^{j-i+1}}=\frac{(-d)^{j-i}}{c^{j-i+1}}, & j \geq i, \\ 0, & j<i .\end{cases}
$$

Proof. Let $c_{i, j}=\sum_{k=1}^{n-2} g_{i, k} g_{k, j}^{\prime}$. Distinctly, $c_{i, j}=0$ for $j<i$. When $i=j$, we obtain

$$
c_{i, i}=g_{i, i} g_{i, i}^{\prime}=-d \cdot \frac{1}{-d}=1 .
$$


For $j \geq i+1$, we obtain

$$
\begin{aligned}
c_{i, j} & =\sum_{k=1}^{n-2} g_{i, k} g_{k, j}^{\prime}=g_{i, i} g_{i, j}^{\prime}+g_{i, i+1} g_{i+1, j}^{\prime} \\
& =c \cdot \frac{(-d)^{j-i}}{c^{j-i+1}}+d \cdot \frac{(-d)^{j-i-1}}{c^{j-i}}=0 .
\end{aligned}
$$

We verify $\mathfrak{G G}^{-1}=I_{n-2}$, where $I_{n-2}$ is $(n-2) \times(n-2)$ identity matrix. Similarly, we check on $\mathfrak{G}^{-1} \mathfrak{G}=I_{n-2}$. Thus, the proof is completed.

Theorem 7. Let $B_{k, n}=\operatorname{Circ}\left(\mathbb{M}_{k+1}, \mathbb{M}_{k+2}, \ldots, \mathbb{M}_{k+n}\right)$ be a Mersenne circulant matrix. Then one acquires

$$
B_{k, n}^{-1}=\operatorname{Circ}\left(u_{1}, u_{2}, \ldots, u_{n}\right),
$$

where

$$
\begin{aligned}
& u_{1}=\frac{1}{f_{k, n}}+\left(\mathbb{M}_{k+1}-\mu_{k} \mathbb{M}_{k+n}-f_{k, n}\right) \\
& \cdot \frac{-\mathbb{M}_{k+n+1}+3 \mathbb{M}_{k+n}+\mathbb{M}_{k+1}-3 \mathbb{M}_{k}}{2 f_{k, n}\left(\mathbb{M}_{k}-\mathbb{M}_{k+n}\right)^{2}} \\
& +\frac{\left(\mathbb{M}_{k+n}-\mu_{k} \mathbb{M}_{k+n-1}\right)}{f_{k, n}\left(\mathbb{M}_{k}-\mathbb{M}_{k+n}\right)} \\
& u_{2}=\frac{2^{k}-f_{k, n} \mathbb{M}_{k+1}}{\mathbb{M}_{k+1} f_{k, n}\left(\mathbb{M}_{k}-\mathbb{M}_{k+n}\right)} \text {, } \\
& u_{3}=\frac{\mathbb{M}_{k+1}-\mu_{k} \mathbb{M}_{k+n}-f_{k, n}}{f_{k, n}} \\
& \cdot \frac{\left(\mathbb{M}_{k+1}-\mathbb{M}_{k+n+1}\right)^{n-3}}{\left[2\left(\mathbb{M}_{k}-\mathbb{M}_{k+n}\right)\right]^{n-2}}+\frac{1}{f_{k, n}} \\
& \cdot \sum_{i=4}^{n}\left(\mathbb{M}_{k+n+4-i}-\mu_{k} \mathbb{M}_{k+n+3-i}\right) \\
& \cdot \frac{\left(\mathbb{M}_{k+1}-\mathbb{M}_{k+n+1}\right)^{n-i}}{\left[2\left(\mathbb{M}_{k}-\mathbb{M}_{k+n}\right)\right]^{n-i+1}}, \\
& u_{4}=\frac{\mathbb{M}_{k+n+2}-\mathbb{M}_{k+2}}{f_{k, n}} \\
& \times\left[\left(\mathbb{M}_{k+1}-\mu_{k} \mathbb{M}_{k+n}-f_{k, n}\right) \cdot \frac{\left(\mathbb{M}_{k+1}-\mathbb{M}_{k+n+1}\right)^{n-4}}{\left[2\left(\mathbb{M}_{k}-\mathbb{M}_{k+n}\right)\right]^{n-2}}\right. \\
& +\sum_{i=4}^{n}\left(\mathbb{M}_{k+n+4-i}-\mu_{k} \mathbb{M}_{k+n+3-i}\right) \\
& \left.\cdot \frac{\left(\mathbb{M}_{k+1}-\mathbb{M}_{k+n+1}\right)^{n-i-1}}{\left[2\left(\mathbb{M}_{k}-\mathbb{M}_{k+n}\right)\right]^{n-i+1}}\right], \\
& u_{s}=0 \quad(s=5,6, \ldots, n) \text {, }
\end{aligned}
$$

where

$$
\begin{gathered}
\mu_{k}=\frac{\mathbb{M}_{k+2}}{\mathbb{M}_{k+1}}, \\
f_{k, n}=\sum_{i=1}^{n-2}\left(\mathbb{M}_{i+k+2}-\mu_{k} \mathbb{M}_{i+k+1}\right) x^{n-i-1} \\
+\mathbb{M}_{k+1}-\mu_{k} \mathbb{M}_{k+n} .
\end{gathered}
$$

Proof. Let

$$
\Pi_{2}=\left(\begin{array}{cccccc}
1 & -\frac{f_{k, n}^{\prime}}{\mathbb{M}_{k+1}} & x_{3} & x_{4} & \cdots & x_{n} \\
0 & 1 & y_{3} & y_{4} & \cdots & y_{n} \\
0 & 0 & 1 & 0 & \cdots & 0 \\
0 & 0 & 0 & 1 & \cdots & 0 \\
\vdots & \vdots & \vdots & \vdots & \ddots & \vdots \\
0 & 0 & 0 & 0 & \cdots & 1
\end{array}\right),
$$

where

$$
\begin{gathered}
\mu_{k}=\frac{\mathbb{M}_{k+2}}{\mathbb{M}_{k+1}}, \\
x_{3}=\frac{\mathbb{M}_{k+n}}{\mathbb{M}_{k+1}}+\frac{f_{k, n}^{\prime}}{f_{k, n}} \cdot \frac{\left(\mu_{k} \mathbb{M}_{k+n}-\mathbb{M}_{k+1}\right)}{\mathbb{M}_{k+1}}, \\
y_{3}=\frac{\mathbb{M}_{k+1}-\mu_{k} \mathbb{M}_{k+n}}{f_{k, n}}, \\
x_{i}=\frac{\mathbb{M}_{k+n+3-i}}{\mathbb{M}_{k+1}+\frac{f_{k, n}^{\prime}}{f_{k, n}}} \\
\cdot \frac{\mu_{k} \mathbb{M}_{k+n+3-i}-\mathbb{M}_{k+n+4-i}}{\mathbb{M}_{k+1}} \\
y_{i}=\frac{(i=4, \ldots, n),}{\mathbb{M}_{k+n+4-i}-\mu_{k} \mathbb{M}_{k+n+3-i}} \\
f_{k, n} \\
f_{k, n}^{\prime}=\sum_{i=1}^{n-1} \mathbb{M}_{i+k+1}\left[\begin{array}{l}
\frac{2(i=4, \ldots, n),}{\left.\mathbb{M}_{k+n+1}-\mathbb{M}_{k+n}-\mathbb{M}_{k}\right)} \\
f_{k, n}^{n-2}\left(\mathbb{M}_{i+k+2}-\mu_{k} \mathbb{M}_{i+k+1}\right) x^{n-i-1} \\
i=1
\end{array},\right.
\end{gathered}
$$

We have

$$
\Gamma B_{k, n} \Pi_{1} \Pi_{2}=\mathscr{D}_{1} \oplus \mathfrak{G},
$$

where $\mathscr{D}_{1}=\operatorname{diag}\left(\mathbb{M}_{k+1}, f_{k, n}\right)$ is a diagonal matrix, and $\mathscr{D}_{1} \oplus \mathfrak{G}$ is the direct sum of $\mathscr{D}_{1}$ and $\mathfrak{G}$. If we denote $\Pi=\Pi_{1} \Pi_{2}$, then we obtain

$$
B_{k, n}^{-1}=\Pi\left(\mathscr{D}_{1}^{-1} \oplus \mathfrak{G}^{-1}\right) \Gamma
$$


Let $B_{k, n}^{-1}=\operatorname{Circ}\left(u_{1}, u_{2}, \ldots, u_{n}\right)$. Since the last row elements of the matrix $\Pi$ are $0,1, y_{3}-1, y_{4}, \ldots, y_{n-1}, y_{n}$, according to Lemma 6 , then the last row elements of $B_{k, n}^{-1}$ are given by the following equations:

$$
\begin{aligned}
u_{2}= & -\frac{\mu_{k}}{f_{k, n}}+\frac{2\left(y_{3}-1\right)}{c}, \\
u_{3}= & \left(y_{3}-1\right) \cdot \frac{(-d)^{n-3}}{c^{n-2}}+\sum_{i=4}^{n} y_{i} \cdot \frac{(-d)^{n-i}}{c^{n-i+1}}, \\
u_{4}= & \left(y_{3}-1\right) \cdot\left[\frac{(-d)^{n-4}}{c^{n-3}}-\frac{3(-d)^{n-3}}{c^{n-2}}\right] \\
& +\sum_{i=4}^{n} y_{i} \cdot\left[\frac{(-d)^{n-i-1}}{c^{n-i}}-\frac{3(-d)^{n-i}}{c^{n-i+1}}\right] \\
= & \left(y_{3}-1\right) \cdot \frac{(-d)^{n-4}}{c^{n-2}}(c+3 d) \\
& +\sum_{i=4}^{n} y_{i} \cdot \frac{(-d)^{n-i-1}}{c^{n-i+1}}(c+3 d) \\
u_{s}= & \left(y_{3}-1\right) \\
& \cdot\left[\frac{(-d)^{n-s}}{c^{n-s+1}}-\frac{3(-d)^{n-s+1}}{c^{n-s+2}}+\frac{2(-d)^{n-s+2}}{c^{n-s+3}}\right] \\
& +\sum_{i=4}^{n-s+5} y_{i} \cdot\left[\frac{(-d)^{n-i-s+3}}{c^{n-i-s+4}}\right. \\
u_{1} & \frac{1}{f_{k, n}}+\frac{-2 d-3 c}{c^{2}}\left(y_{3}-1\right)+\frac{2}{c} y_{4}, \\
= & \left.\left(y_{3}-1\right) \cdot \frac{(-d)^{n-s}}{c^{n-s+3}}+\sum_{i=4}^{n-s+5} y_{i} \cdot \frac{(-d)^{n-i-s+3}}{c^{n-i-s+6}}\right] \\
c^{n-i-s+5} & (c+2 d)(c+d) \quad\left(s=5,6, \ldots, n ; t<0,(-d)^{t}=0\right), \\
& {\left[(-d)^{n-i-s+5}\right] }
\end{aligned}
$$

where $d=\mathbb{M}_{k+n+1}-\mathbb{M}_{k+1}, c=2\left(\mathbb{M}_{k}-\mathbb{M}_{k+n}\right)$, according to Lemma 1; then we have

(i) $c+d=0$,

(ii) $c+2 d=2^{k+n+1}-2^{k+1}$.

We get

$$
\begin{aligned}
u_{1}= & \frac{1}{f_{k, n}}+\left(\mathbb{M}_{k+1}-\mu_{k} \mathbb{M}_{k+n}-f_{k, n}\right) \\
& \cdot \frac{-\mathbb{M}_{k+n+1}+3 \mathbb{M}_{k+n}+\mathbb{M}_{k+1}-3 \mathbb{M}_{k}}{2 f_{k, n}\left(\mathbb{M}_{k}-\mathbb{M}_{k+n}\right)^{2}} \\
& +\frac{\left(\mathbb{M}_{k+n}-\mu_{k} \mathbb{M}_{k+n-1}\right)}{f_{k, n}\left(\mathbb{M}_{k}-\mathbb{M}_{k+n}\right)},
\end{aligned}
$$

$$
\begin{aligned}
& u_{2}=\frac{2^{k}-f_{k, n} \mathbb{M}_{k+1}}{\mathbb{M}_{k+1} f_{k, n}\left(\mathbb{M}_{k}-\mathbb{M}_{k+n}\right)}, \\
& u_{3}=\frac{\mathbb{M}_{k+1}-\mu_{k} \mathbb{M}_{k+n}-f_{k, n}}{f_{k, n}} \\
& \cdot \frac{\left(\mathbb{M}_{k+1}-\mathbb{M}_{k+n+1}\right)^{n-3}}{\left[2\left(\mathbb{M}_{k}-\mathbb{M}_{k+n}\right)\right]^{n-2}}+\frac{1}{f_{k, n}} \\
& \cdot \sum_{i=4}^{n}\left(\mathbb{M}_{k+n+4-i}-\mu_{k} \mathbb{M}_{k+n+3-i}\right) \\
& \cdot \frac{\left(\mathbb{M}_{k+1}-\mathbb{M}_{k+n+1}\right)^{n-i}}{\left[2\left(\mathbb{M}_{k}-\mathbb{M}_{k+n}\right)\right]^{n-i+1}} \\
& u_{4}=\frac{\mathbb{M}_{k+n+2}-\mathbb{M}_{k+2}}{f_{k, n}} \\
& \times\left[\left(\mathbb{M}_{k+1}-\mu_{k} \mathbb{M}_{k+n}-f_{k, n}\right) \frac{\left(\mathbb{M}_{k+1}-\mathbb{M}_{k+n+1}\right)^{n-4}}{\left[2\left(\mathbb{M}_{k}-\mathbb{M}_{k+n}\right)\right]^{n-2}}\right. \\
& +\sum_{i=4}^{n}\left(\mathbb{M}_{k+n+4-i}-\mu_{k} \mathbb{M}_{k+n+3-i}\right) \\
& \left.\cdot \frac{\left(\mathbb{M}_{k+1}-\mathbb{M}_{k+n+1}\right)^{n-i-1}}{\left[2\left(\mathbb{M}_{k}-\mathbb{M}_{k+n}\right)\right]^{n-i+1}}\right], \\
& u_{s}=0 \quad(s=5,6, \ldots, n) \text {. }
\end{aligned}
$$

Thus, the proof is completed.

\section{Determinants and Inverses of Fermat and Mersenne Left Circulant Matrix}

In this section, let $A_{k, n}^{\prime}=\operatorname{LCirc}\left(\mathbb{F}_{k+1}, \mathbb{F}_{k+2}, \ldots, \mathbb{F}_{k+n}\right)$ and $B_{k, n}^{\prime}=\operatorname{LCirc}\left(\mathbb{M}_{k+1}, \mathbb{M}_{k+2}, \ldots, \mathbb{M}_{k+n}\right)$ be Mersenne and Fermat left circulant matrices, respectively. By using the obtained conclusions, we give a determinant formula for the matrix $A_{k, n}^{\prime}$ and $B_{k, n}^{\prime}$. In addition, the inverse matrices of $A_{k, n}^{\prime}$ and $B_{k, n}^{\prime}$ are derived.

According to Lemma 2 in [14] and Theorems 2, 4, 5, and 7 , we can obtain the following theorems.

Theorem 8. Let $A_{k, n}^{\prime}=\operatorname{LCirc}\left(\mathbb{F}_{k+1}, \mathbb{F}_{k+2}, \ldots, \mathbb{F}_{k+n}\right)$ be a Fermat left circulant matrix; then one has

$$
\begin{aligned}
\operatorname{det} A_{k, n}^{\prime}= & (-1)^{(n-1)(n-2) / 2} \cdot \mathbb{F}_{k+1} \\
& \cdot\left[\sum_{j=1}^{n-2}\left(\mathbb{F}_{j+k+2}-\tau_{k} \mathbb{F}_{j+k+1}\right) p^{n-j-1}+\mathbb{F}_{k+1}-\tau_{k} \mathbb{F}_{k+n}\right] \\
& \cdot\left(\mathbb{F}_{k+1}-\mathbb{F}_{k+n+1}\right)^{n-2},
\end{aligned}
$$

where $\tau_{k}=\mathbb{F}_{k+2} / \mathbb{F}_{k+1}, p=2\left(\mathbb{F}_{k+n}-\mathbb{F}_{k}\right) /\left(\mathbb{F}_{k+n+1}-\mathbb{F}_{k+1}\right)$, and $\mathbb{F}_{k+n}$ is the $(k+n)$ th Fermat number. Moreover, $A_{k, n}^{\prime}$ is singular if and 
only if $\left(1-\alpha \kappa_{l}\right)\left(1-\beta \kappa_{l}\right) \neq 0$ and $\mathbb{F}_{k+1}-2 \kappa_{l} \mathbb{F}_{k}-\mathbb{F}_{k+n+1}+2 \kappa_{l} \mathbb{F}_{k+n}=$ 0 , for $k \in N, n \in N_{+}$, where $\kappa_{l}=\cos (2 l \pi / n)+i \sin (2 l \pi / n)$, $l=1,2, \ldots, n$.

Theorem 9. Let $A_{k, n}^{\prime}=\operatorname{LCirc}\left(\mathbb{F}_{k+1}, \mathbb{F}_{k+2}, \ldots, \mathbb{F}_{k+n}\right)$ be a Fermat left circulant matrix; then

$$
\begin{aligned}
\left(A_{k, n}^{\prime}\right)^{-1} & =\operatorname{Circ}^{-1}\left(\mathbb{F}_{k+1}, \mathbb{F}_{k+2}, \ldots, \mathbb{F}_{k+n}\right) \cdot \Delta \\
& =\operatorname{Circ}\left(v_{1}, v_{2}, \ldots, v_{n}\right) \cdot \Delta \\
& =\operatorname{LCirc}\left(v_{1}, v_{n}, \ldots, v_{2}\right),
\end{aligned}
$$

where $v_{1}, v_{2}, \ldots, v_{n}$ were given by Theorem 4 and $\Delta=$ $\operatorname{LCirc}(1,0, \ldots, 0)$ was given by Lemma 2 in [14].

Theorem 10. Let $B_{k, n}^{\prime}=\operatorname{LCirc}\left(\mathbb{M}_{k+1}, \mathbb{M}_{k+2}, \ldots, \mathbb{M}_{k+n}\right)$ be a Mersenne left circulant matrix; then one has

$$
\begin{aligned}
& \operatorname{det} B_{k, n}^{\prime}=(-1)^{(n-1)(n-2) / 2} \cdot \mathbb{M}_{k+1} \\
& \cdot\left[\mathbb{M}_{k+1}-\mu_{k} \mathbb{M}_{k+n}\right. \\
& \left.+\sum_{j=1}^{n-2}\left(\mathbb{M}_{j+k+2}-\mu_{k} \mathbb{M}_{j+k+1}\right) z^{n-j-1}\right] \\
& \cdot\left(\mathbb{M}_{k+1}-\mathbb{M}_{k+n+1}\right)^{n-2} \text {, }
\end{aligned}
$$

where $\mu_{k}=\mathbb{M}_{k+2} / \mathbb{M}_{k+1}, z=2\left(\mathbb{M}_{k+n}-\mathbb{M}_{k}\right) /\left(\mathbb{M}_{k+n+1}-\mathbb{M}_{k+1}\right)$, and $\mathrm{M}_{k+n}$ is the $(k+n)$ th Mersenne number. Furthermore, $B_{k, n}^{\prime}$ is singular if and only if $\left(1-\alpha \kappa_{l}\right)\left(1-\beta \kappa_{l}\right) \neq 0$ and $\mathbb{M}_{k+1}-$ $2 \kappa_{l} \mathbb{M}_{k}-\mathbb{M}_{k+n+1}+2 \kappa_{l} \mathbb{M}_{k+n}=0$, for $k \in N, n \in N_{+}$, where $\kappa_{l}=\cos (2 l \pi / n)+i \sin (2 l \pi / n), l=1,2, \ldots, n$.

Theorem 11. Let $B_{k, n}^{\prime}=\operatorname{LCirc}\left(\mathbb{M}_{k+1}, \mathbb{M}_{k+2}, \ldots, \mathbb{M}_{k+n}\right)$ be a Mersenne left circulant matrix; then one has

$$
\begin{aligned}
\left(B_{k, n}^{\prime}\right)^{-1} & =\operatorname{Circ}^{-1}\left(\mathbb{M}_{k+1}, \mathbb{M}_{k+2}, \ldots, \mathbb{M}_{k+n}\right) \cdot \Delta \\
& =\operatorname{Circ}\left(u_{1}, u_{2}, \ldots, u_{n}\right) \cdot \Delta \\
& =\operatorname{LCirc}\left(u_{1}, u_{n}, \ldots, u_{2}\right)
\end{aligned}
$$

where $u_{1}, u_{2}, \ldots, u_{n}$ were given by Theorem 7 and $\Delta=$ LCirc $(1,0, \ldots, 0)$ was given by Lemma 2 in [14].

\section{Conclusion}

In this paper, we present the exact determinants and the inverse matrices of Fermat and Mersenne circulant matrix, respectively. Furthermore, we give the exact determinants and the inverse matrices of Fermat and Mersenne left circulant matrix. Meanwhile, the nonsingularity of these special matrices is discussed. On the basis of circulant matrices technology, we will develop solving the problems in [19-22].

\section{Conflict of Interests}

The authors declare that there is no conflict of interests regarding the publication of this paper.

\section{Acknowledgment}

This work was supported by the GRRC Program of Gyeonggi Province ((GRRC SUWON 2014-B4), Development of Cloud Computing-Based Intelligent Video Security Surveillance System with Active Tracking Technology). Their support is gratefully acknowledged.

\section{References}

[1] C. Zhang, G. Dangelmayr, and I. Oprea, "Storing cycles in Hopfield-type networks with pseudoinverse learning rule: admissibility and network topology," Neural Networks, vol. 46, pp. 283-298, 2013.

[2] D. Rocchesso and J. O. Smith, "Circulant and elliptic feedback delay networks for artificial reverberation," IEEE Transactions on Speech and Audio Processing, vol. 5, no. 1, pp. 51-63, 1997.

[3] Y. Jing and H. Jafarkhani, "Distributed differential space-time coding for wireless relay networks," IEEE Transactions on Communications, vol. 56, no. 7, pp. 1092-1100, 2008.

[4] M. Bašić, "Characterization of quantum circulant networks having perfect state transfer," Quantum Information Processing, vol. 12, no. 1, pp. 345-364, 2013.

[5] G. Wang, F. Gao, Y.-C. Wu, and C. Tellambura, "Joint CFO and channel estimation for OFDM-based two-way relay networks," IEEE Transactions on Wireless Communications, vol. 10, no. 2, pp. 456-465, 2011.

[6] J. Li, J. Yuan, R. Malaney, M. Xiao, and W. Chen, "Fulldiversity binary frame-wise network coding for multiplesource multiple-relay networks over slow-fading channels," IEEE Transactions on Vehicular Technology, vol. 61, no. 3, pp. 1346-1360, 2012.

[7] P. J. Davis, Circulant Matrices, John Wiley \& Sons, New York, NY, USA, 1979.

[8] Z. L. Jiang and Z. X. Zhou, Circulant Matrices, Chengdu Technology University Publishing Company, Chengdu, China, 1999.

[9] Z. Jiang, "On the minimal polynomials and the inverses of multilevel scaled factor circulant matrices," Abstract and Applied Analysis, vol. 2014, Article ID 521643, 10 pages, 2014.

[10] Z. Jiang, T. Xu, and F. Lu, "Isomorphic operators and functional equations for the skew-circulant algebra," Abstract and Applied Analysis, vol. 2014, Article ID 418194, 8 pages, 2014.

[11] J. Li, Z. L. Jiang, and F. L. Lu, "Determinants, norms, and the spread of circulant matrices with Tribonacci and generalized Lucas numbers," Abstract and Applied Analysis, vol. 2014, Article ID 381829, 9 pages, 2014.

[12] Z. L. Jiang, "Nonsingularity of two classes of cyclic matrices," Mathematics in Practice and Theory, no. 2, pp. 52-58, 1995.

[13] J.-J. Yao and Z.-L. Jiang, "The determinants, inverses, norm, and spread of skew circulant type matrices involving any continuous Lucas numbers," Journal of Applied Mathematics, vol. 2014, Article ID 239693, 10 pages, 2014. 
[14] Z. Jiang, Y. Gong, and Y. Gao, "Invertibility and explicit inverses of circulant-type matrices with $k$-Fibonacci and $k$ Lucas numbers," Abstract and Applied Analysis, vol. 2014, Article ID 238953, 9 pages, 2014.

[15] L. Dazheng, "Fibonacci-Lucas quasi-cyclic matrices," The Fibonacci Quarterly, vol. 40, no. 3, pp. 280-286, 2002.

[16] D. Bozkurt and T.-Y. Tam, "Determinants and inverses of circulant matrices with JACobsthal and JACobsthal-Lucas Numbers," Applied Mathematics and Computation, vol. 219, no. 2, pp. 544-551, 2012.

[17] A. F. Horadam, "Further appearence of the Fibonacci sequence," The Fibonacci Quarterly, vol. 1, no. 4, pp. 41-42, 1963.

[18] A. İpek and K. Arı, "On Hessenberg and pentadiagonal determinants related with FIBonacci and FIBonacci-like numbers," Applied Mathematics and Computation, vol. 229, pp. 433-439, 2014.

[19] H. Dong, Z. Wang, and H. Gao, "Distributed $H_{\infty}$ filtering for a class of markovian jump nonlinear time-delay systems over lossy sensor networks," IEEE Transactions on Industrial Electronics, vol. 60, no. 10, pp. 4665-4672, 2013.

[20] Z. Wang, H. Dong, B. Shen, and H. Gao, "Finite-horizon $H_{\infty}$ filtering with missing measurements and quantization effects," IEEE Transactions on Automatic Control, vol. 58, no. 7, pp. 17071718, 2013.

[21] D. Ding, Z. Wang, J. Hu, and H. Shu, "Dissipative control for state-saturated discrete time-varying systems with randomly occurring nonlinearities and missing measurements," International Journal of Control, vol. 86, no. 4, pp. 674-688, 2013.

[22] J. Hu, Z. Wang, B. Shen, and H. Gao, "Quantised recursive filtering for a class of nonlinear systems with multiplicative noises and missing measurements," International Journal of Control, vol. 86, no. 4, pp. 650-663, 2013. 


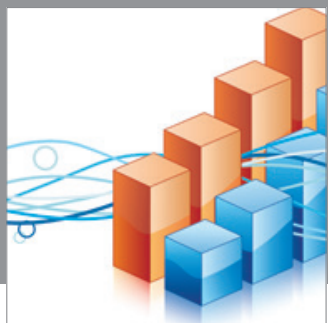

Advances in

Operations Research

mansans

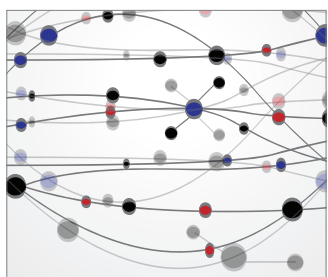

The Scientific World Journal
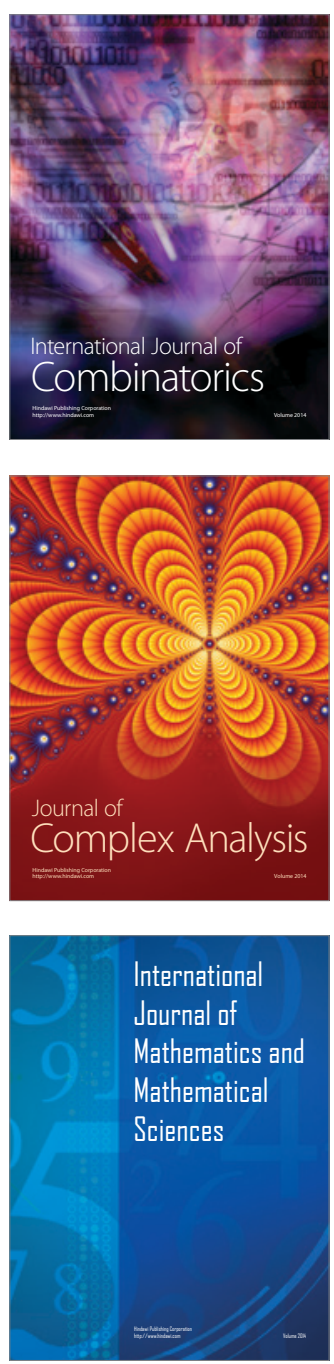
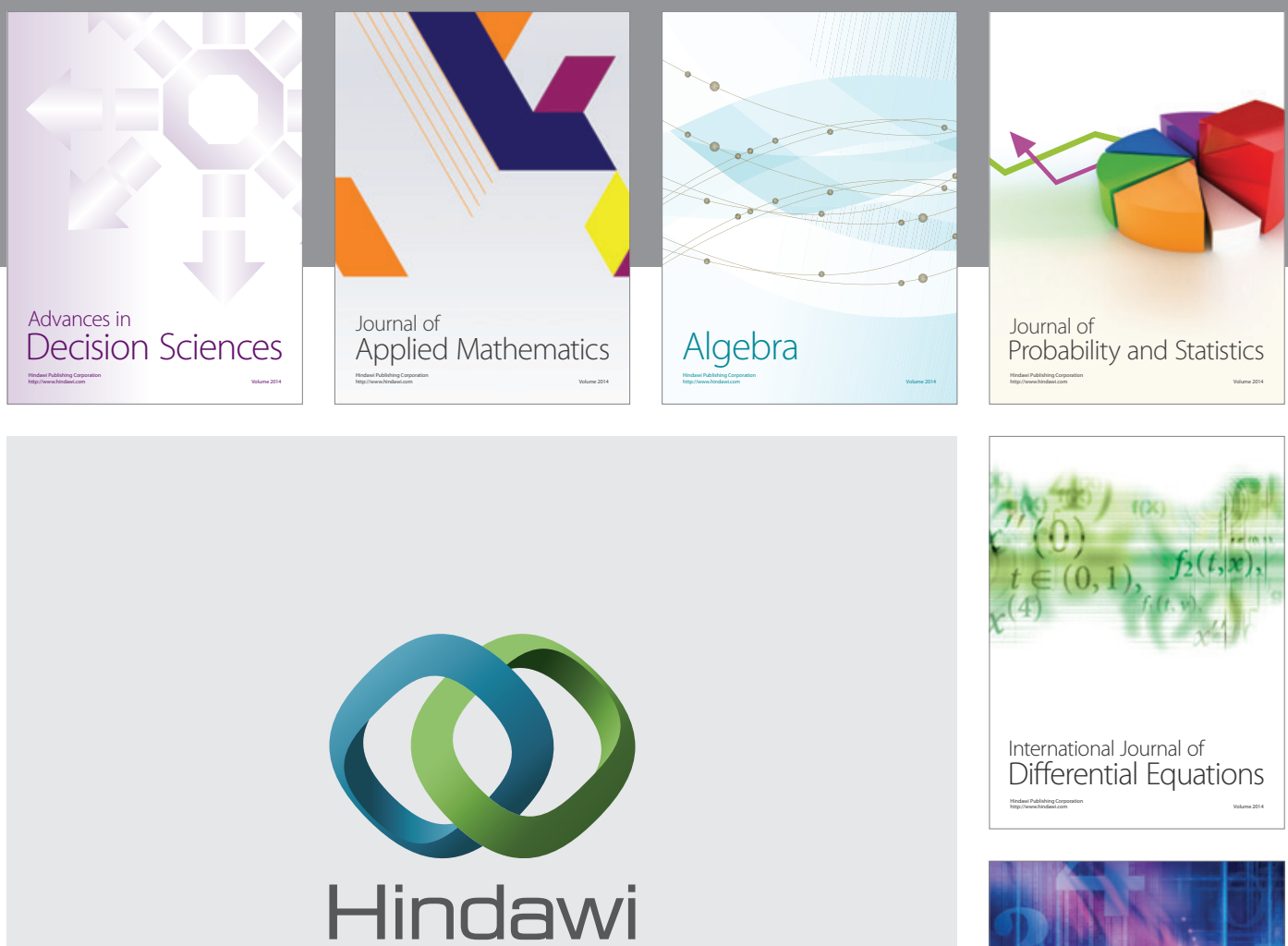

Submit your manuscripts at http://www.hindawi.com
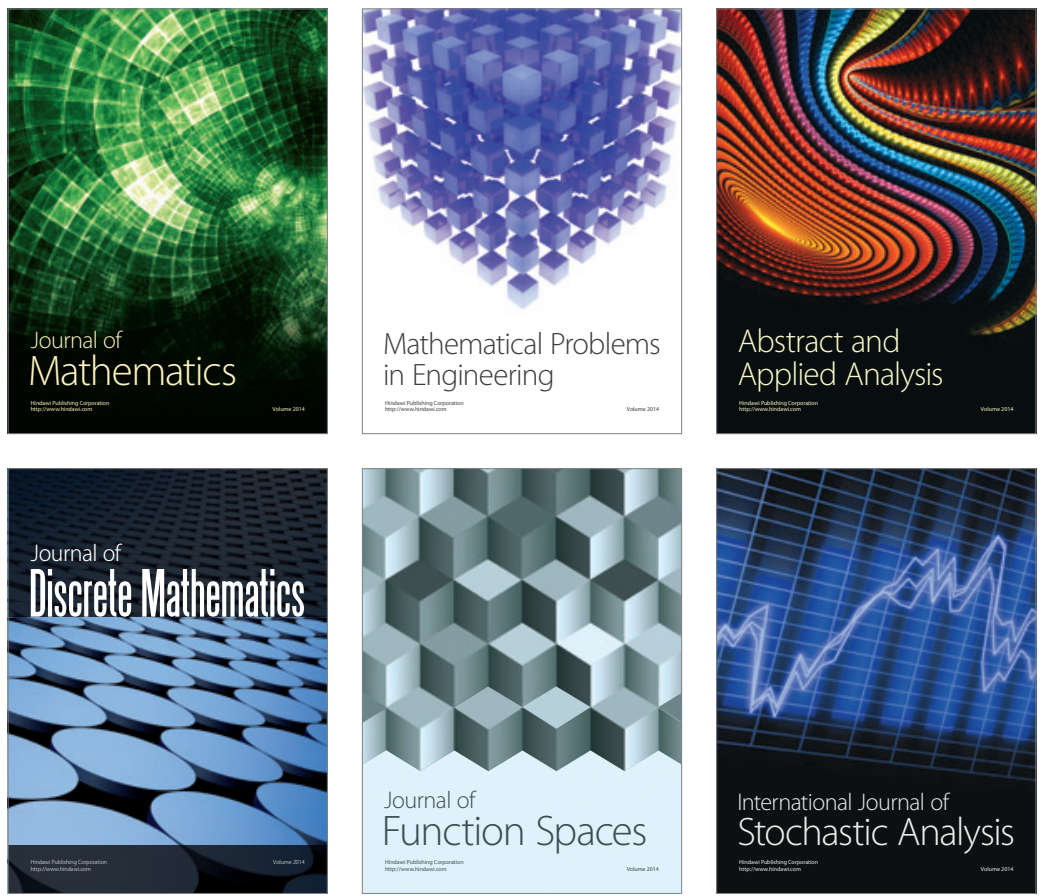

Journal of

Function Spaces

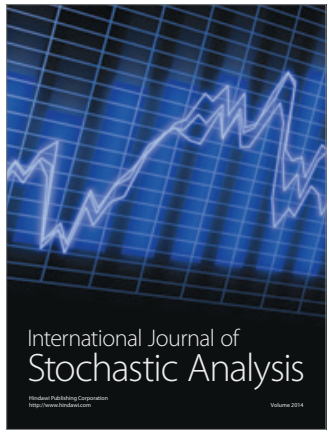

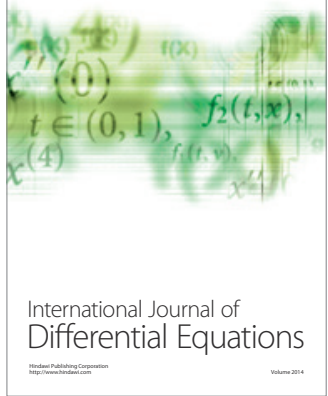
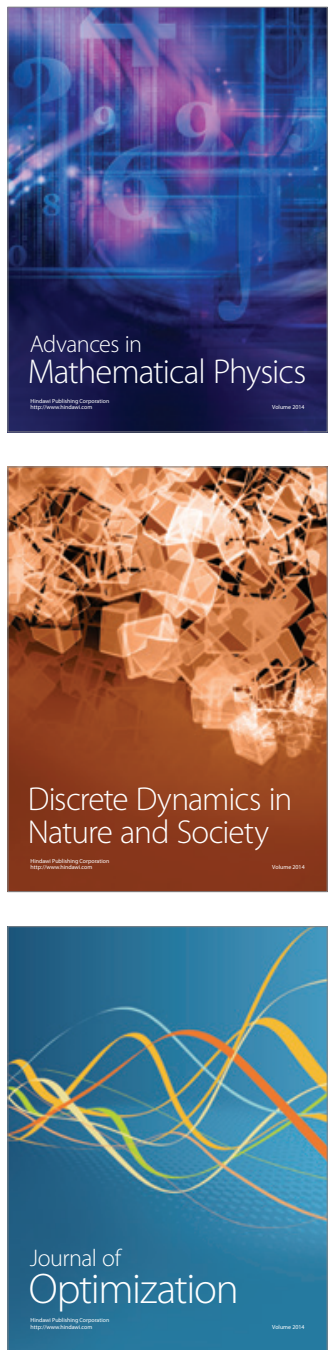